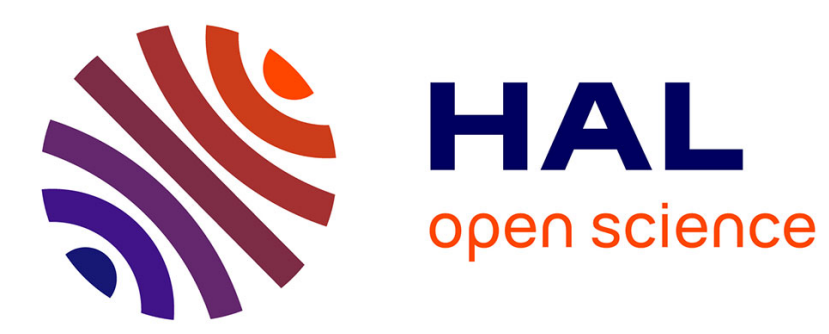

\title{
Experimental route to chaos of an external-cavity semiconductor laser
}

\author{
Byungchil Kim, A. Locquet, Daeyoung Choi, D S Citrin
}

\section{To cite this version:}

Byungchil Kim, A. Locquet, Daeyoung Choi, D S Citrin. Experimental route to chaos of an externalcavity semiconductor laser. Physical Review A : Atomic, molecular, and optical physics [1990-2015], 2015, 91, 10.1103/physreva.91.061802 . hal-02993518

\section{HAL Id: hal-02993518 https://hal.science/hal-02993518}

Submitted on 6 Nov 2020

HAL is a multi-disciplinary open access archive for the deposit and dissemination of scientific research documents, whether they are published or not. The documents may come from teaching and research institutions in France or abroad, or from public or private research centers.
L'archive ouverte pluridisciplinaire HAL, est destinée au dépôt et à la diffusion de documents scientifiques de niveau recherche, publiés ou non, émanant des établissements d'enseignement et de recherche français ou étrangers, des laboratoires publics ou privés. 


\title{
Experimental route to chaos of an external-cavity semiconductor laser
}

\author{
Byungchil Kim, Alexandre Locquet, Daeyoung Choi, and D. S. Citrin \\ Georgia Institute of Technology, School of Electrical and Computer Engineering, Atlanta, Georgia 30332-0250, USA \\ and UMI 2958 Georgia Tech-CNRS, Georgia Tech Lorraine, 2 Rue Marconi F-57070, Metz, France \\ (Received 26 January 2015; published 5 June 2015)
}

\begin{abstract}
We report experimental bifurcation diagrams of a semiconductor laser, biased well above threshold, subjected to external optical feedback. As feedback is increased, we see a quasiperiodic route to chaos interrupted by several windows of periodicity corresponding to limit cycles, differing in frequency by multiples of the external-cavity free-spectral range that have developed around external-cavity modes (ECMs) whose frequency is slightly larger than that of the solitary laser. Successive windows correspond to the transition between two limit cycles either on the same or neighboring ECMs. For larger feedback, the laser operates in a chaotic regime around numerous negatively shifted external-cavity modes. These experimental observations detail the bifurcations leading to fully developed chaos in this system, and further provide detailed insight on the standard theoretical framework applied to these lasers.
\end{abstract}

DOI: 10.1103/PhysRevA.91.061802

PACS number(s): 42.55.Px, 05.45.-a

In an external-cavity semiconductor lasers (ECL), the light produced by a laser diode (LD) is reinjected into its active layer upon reflection by an external mirror. The externalcavity time of flight $\tau$ creates an optical delay that leads to a high-dimensional dynamical system in which a variety of regimes have been observed and in particular chaos of various complexities [1,2]. The tunability of the behaviors, depending on operating and design parameters, as well as their high-speed dynamics, have given ECLs, especially in the long-cavity case, a prominent role for fundamental studies of nonlinear dynamics [3] as well as for applications such as tunable laser sources [4], physical-layer security [5], light detecting and ranging (LIDAR) [6], high-speed random number generation [7], and reservoir computing [8].

Understanding the sensitivity of LDs to optical feedback is of fundamental importance to achieve an adequate understanding of the route to chaos in ECLs as well as more generally for control of ECL dynamics for applications. Consequently, ECL dynamics have been widely investigated [1-3,9-12], typically analyzed with the Lang and Kobayashi (LK) model [13]. On its basis, numerous authors have plotted bifurcation diagrams (BD) $[3,14-17]$ as a BD is of utmost importance in the dynamical-system analysis. These theoretical BDs show a complex sequence of bifurcations, characteristic of highdimensional systems, in which the route to fully developed chaos is typically interrupted by windows of periodic or quasiperiodic behaviors and/or by a switch to a different attractor or set of attractors. Surprisingly, experimental support of those theoretical results is sparse. Contrary to the LK model, some experiments have reported simpler routes to chaos: quasiperiodic [14], period doubling [18], and subharmonic cascaded bifurcation [19], typical of low-dimensional systems [20]. These claims are based on the observation, usually relying on a spectral analysis, of a succession of dynamical regimes for a few discrete parameter values rather than for a continuously tuned parameter. Thus a complete, experimentally verified understanding of the dynamical regimes of an ECL, and of the bifurcations between them, has not yet been attained.

Recently, we reported an experimental, partly resolved BD [21,22]. Injection current $I$ just above threshold $I_{\text {th }}$ was used, for which spontaneous-emission noise obscures the details of the bifurcations, and thus reliable identifications were not easily made.

In this Rapid Communicaiton, we consider the high$I$ case where spontaneous-emission noise plays a smaller role. We show fully resolved BDs of an ECL biased well above $I_{\text {th }}$ and unambiguously identify the bifurcation cascade leading to chaos. Thus, we report a conclusive experimental demonstration of a full route to chaos in a Distributed feedback laser (DFB) ECL initiating with a Hopf bifurcation. The LD is biased at $I \sim 2-3 I_{t h}$ with external-cavity length $L$ chosen so that the external-cavity free-spectral range (FSR) $f_{\tau}=c /(2 L)$ is lower than, and not a submultiple of, the relaxation-oscillation frequency of the solitary LD $f_{\mathrm{RO}}$. We observe a quasiperiodic route to chaos interrupted by windows of periodicity or quasiperiodicity and emphasize that the first two bifurcations seen are Hopf bifurcations as correctly predicted by the LK model in the common case in which $f_{\mathrm{RO}}$ is not an integer multiple of $f_{\tau}=\tau^{-1}$ [14,17]. Also, in the periodic windows, we observe coexistence of several limit cycles on the same external-cavity mode (ECM) for successive increasing feedback strengths, and on neighboring ECMs, separated in frequency by small multiples of $f_{\tau}$.

The experiment is shown in Fig. 1(a) [22]; $I_{\text {th }}=9.27 \mathrm{~mA}$. Temperature and $I$ are stabilized. An oscilloscope $(12 \mathrm{GHz}$ bandwidth) captures the optical intensity $\mathcal{I}(t)$ emitted by the laser. We also measure the RF spectrum with a spectrum analyzer ( $23 \mathrm{GHz}$ bandwidth) and the optical spectrum with a high-resolution optical spectrum analyzer (APEX AP2440) (10 MHz optical resolution); $L$ is set to $30 \mathrm{~cm}, \tau=2 \mathrm{~ns}$, and $f_{\tau}=500 \mathrm{MHz}$ with $f_{R O}$ in the multi-GHz range. The experimental feedback strength $\eta$ is proportional to the optical intensity fed back into the LD; $\eta$ is varied via the angle of the quarter-wave plate (QWP) in the feedback loop. We control the QWP angle in $0.01^{\circ}$ increments, giving 4500 possible different $\eta$ values with a motorized rotation stage under high-stability conditions. Maximum feedback $(\eta=1)$ is when the QWP angle is such that the electric field is not subjected to any rotation. In that case, the percentage of threshold reduction $\left(I_{t h}-I\right) / I_{t h}$ is $\sim 0.066$ and $\sim 20 \%$ of the optical power is fed back onto the collimating lens. 
(a)

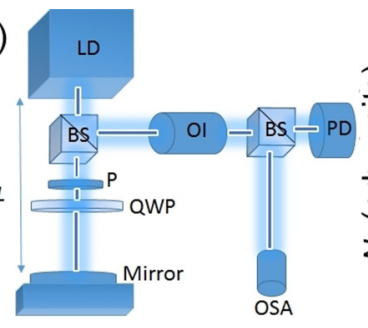

FIG. 1. (Color online) (a) Experimental setup. LD, laser diode; BS, beam splitter; PD, photodetector; P, polarizer; QWP, quarter-wave plate; OI, optical isolator; OSA, optical spectrum analyzer; and $L$, external-cavity length. $\mathrm{LD}$ is an intrinsically single-longitudinal mode InGaAsP DFB laser at $1550 \mathrm{~nm}$ with maximum power of $15 \mathrm{~mW}$. (b) Ellipse structure of fixed points in $\Delta \phi$-vs- $N$ plane. Circles represent ECMs; crosses represent antimodes. Labels indicate mode number.

The experimental results are analyzed and compared with the LK model, which is widely used to describe the effect of optical feedback and has proven successful in predicting many aspects of the dynamics $[9,10,16,23]$, though some inconsistencies persist [24]. Still, due to its considerable success we use the LK to analyze our experiments. According to LK, as feedback is increased, steady-state CW solutions appear in pairs as a result of saddle-node bifurcations: ECMs and antimodes that are unstable saddle points $[9,12]$. These may be thought of as the nonlinear modes of the ECL. The ECMs and antimodes are known to lie on an ellipse in the plane formed by the carrier density (inversion) $N(t)$ versus phase difference $\Delta \phi(t)=\phi(t)-\phi(t-\tau)$ plane, with $\phi$ the slowly varying phase of the electric field, as shown in Fig. 1(b) [25]. Two ECMs stand out: the minimum linewidth mode (MLM) and the maximum gain mode (MGM). The MGM has the lowest frequency (high-gain end) and is always stable $[9,26,27]$. The MLM is the closest ECM in frequency to the solitary LD mode. ECL dynamics can be visualized in terms of the trajectory moving among the ECMs and antimodes on this ellipse. Simulations of the LK model [3,14-17] predict complex routes to chaos which typically start, when $f_{R O}$ is not an integer multiple of $f_{\tau}$, as quasiperiodic routes but which are also typically interrupted by windows corresponding to periodicity, quasiperiodicity, or attractor switching.

Figure 2(a) shows the experimental $\mathrm{BD}$ (ramping $\eta$ up) with $I=22.08 \mathrm{~mA}$ and $L=30 \mathrm{~cm}$, giving $f_{\mathrm{RO}} \sim 7.4 \mathrm{GHz}$ and $f_{\tau} \sim 500 \mathrm{MHz}$. This BD is obtained by taking the local extrema of $\mathcal{I}(t)$ at each $\eta$. Density is high in white but low in black regions. The corresponding time-averaged voltage drop across the injection leads of the LD $V_{\mathrm{LD}}$ is shown in Fig. 2(b). It has been shown $[28,29]$ that $V_{\mathrm{LD}}$ tracks the changes in $N$. A reduction (increase) in $N$ caused by $\eta$ reduces (increases) the difference between quasi-Fermi levels in the $p$ - and $n$-type quasineutral regions of the LD. Thus, the overall reduction (increase) in $N$ in the active region leads to a reduction (increase) in $V_{\mathrm{LD}}$. Thus $V_{\mathrm{LD}}$ provides information on dynamical variable variable $N$ [29], complementing the more conventionally probed optical intensity.

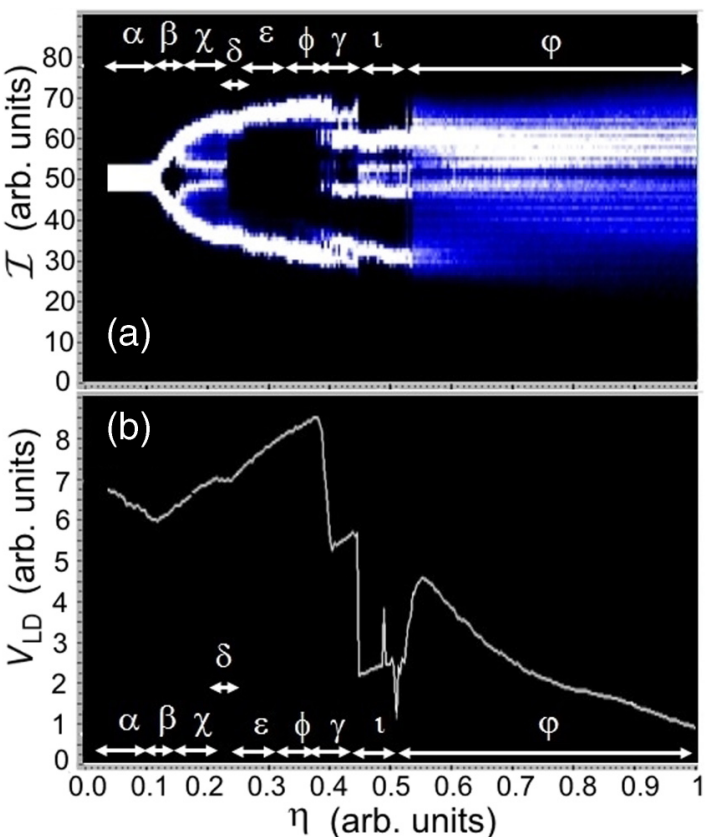

FIG. 2. (Color online) (a) Experimental forward BD for $I=$ $22.08 \mathrm{~mA}$ and $L=30 \mathrm{~cm}$ and (b) corresponding $V_{\mathrm{LD}}$.

To further elucidate the ECL dynamics, experimental RF spectra of $\mathcal{I}(t)$ (left column), optical spectra (right column), and $\mathcal{I}(t)$ (inset) at various $\eta$ are plotted in Fig. 3 for $I=$ $22.08 \mathrm{~mA} ; 0 \mathrm{GHz}$ on the optical-frequency scale corresponds to the ECM at 193.271 THz. We see from Figs. 2 and 3 that the ECL is initially in ECM 1 . In the corresponding region $\alpha(\eta \sim 0.05)$ of Figs. 2(a), 2(b), 3(a1), and 3(a2) only one fixed point (ECM 1) participates in the output, and the ECL displays $\mathrm{CW}$ behavior. From Fig. 2(b), one sees $V_{\mathrm{LD}}$ decrease with increasing $\eta$ in this regime, indicating a decrease in $N$ and an increase in power, consistent with predictions of LK [14].

As $\eta$ is increased above 0.12 , it is clearly seen in the BD and from the shape of $I(t)$ in Fig. 3(b2) that the ECL undergoes periodic oscillation. This oscillation is manifested as sidebands $\pm 8 \mathrm{GHz}$ from ECM 1 , a frequency that also stands out in the RF spectrum [Fig. 3(b1)]. Also, $V_{\mathrm{LD}}$ is monotonically increasing in $\eta$ [region $\beta$ of Figs. 2(a) and 2(b)]. This transition in the dynamics corresponds to a Hopf bifurcation leading to a limit cycle with frequency close to $f_{R O}$, as predicted by LK $[10,30]$.

With increasing $\eta$, additional sidebands near $\pm f_{\tau}$ appear in the optical spectrum [Fig. 3(c2)], revealing the presence of a second frequency in the dynamics. This other frequency is confirmed by the RF spectrum [Fig. 3(c1)], and the corresponding time series of $\mathcal{I}(t)$ exhibits quasiperiodic oscillation. The undamping of a second frequency close to $f_{\tau}$ corresponds to a secondary Hopf bifurcation predicted by LK $[14,17]$ and corresponding to the development, in phase space, of a torus attractor. Note that both the limit cycle and the torus result from the destabilization of ECM 1 and are located around it in phase space. This experimental observation of this sequence of two Hopf bifurcations provides a fundamental point of comparison with the LK model.

For a further increase in $\eta$, another limit cycle appears in the BD. The optical spectrum shows [Fig. 3(d2)] that this 


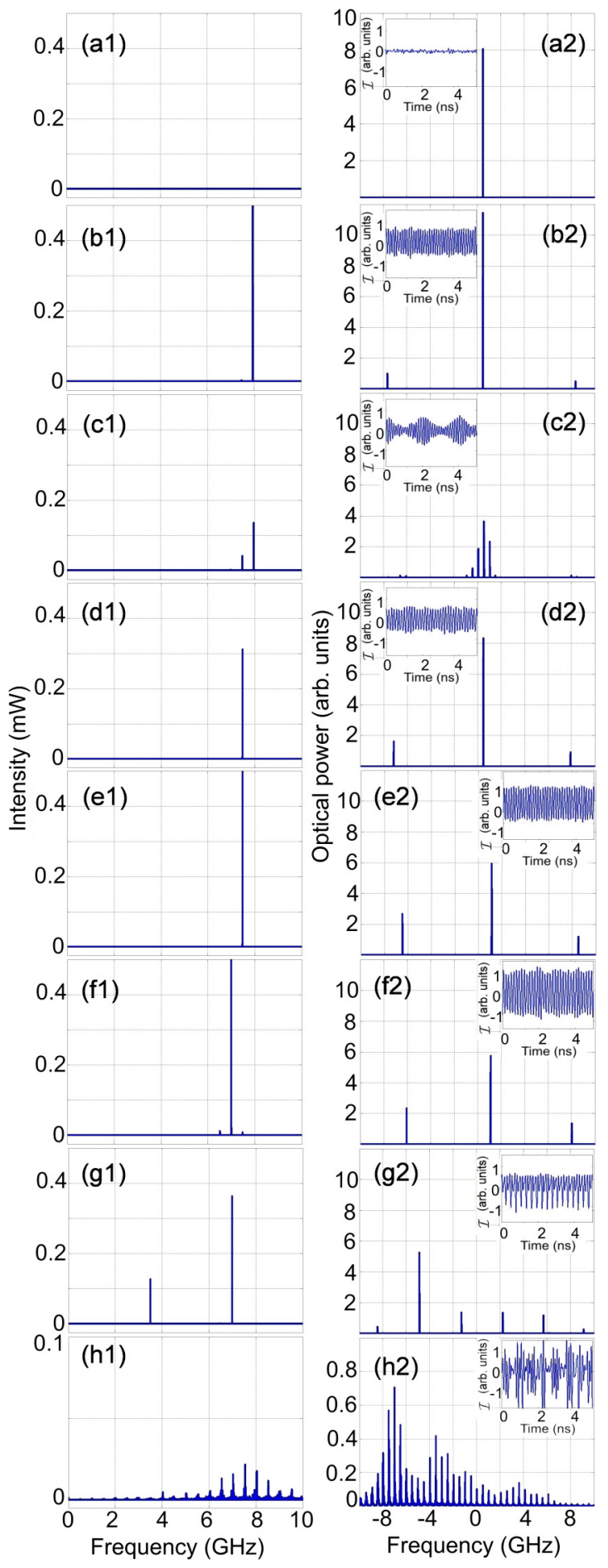

FIG. 3. (Color online) Experimental RF spectra (first column), and optical spectrum with OSA of $\mathcal{I}(t)$ (second column), and associated $\mathcal{I}(t)$ (inset): (a1) and (a2) $\eta=0.05$, (b1) and (b2) 0.13 , (c1) and (c2) 0.2, (d1) and (d2) 0.26, (e1) and (e2) 0.28, (f1) and (f2) $0.35,(\mathrm{~g} 1)$ and (g2) 0.5, and (h1) and (h2) 0.8 .

cycle is still centered on ECM 1 and has now a frequency of approximately $\pm 7.5 \mathrm{GHz}$, evidenced both by the RF [Fig. 3(d1)] and optical spectra [Fig. 3(d2)]. Interestingly, the dominant ECM (1) does not change in between Figs. 3(b2) and $3(\mathrm{~d} 2)$, thus providing experimental evidence of the existence, at different feedback levels, of two periodic solutions with different frequencies, 8 and $7.5 \mathrm{GHz}$, whose separation is $\sim f_{\tau}$. Mork et al. [10], Ritter and Haug [31], and Erneux [32] had demonstrated, based on LK, the existence of two limit cycles originating in the same ECM but differing in frequency by approximately $f_{\tau}$. These previous works show that our observation of several limit cycles with different frequencies around the same ECM is compatible with LK. Pieroux et al. [33] have shown that, more generally, multiple periodic solutions can occur in class-B lasers subjected to delayed feedback and that their interaction can lead to quasiperiodic solutions. As $\eta$ is further increased, and region $\epsilon$ of Figs. 2(a) is reached, one observes a small discontinuity in the $\mathrm{BD}$, as well as a discontinuity in the slope of $V_{\mathrm{LD}}$ [Figs. 2(b)]. The optical spectrum [Figs. 3(e2)] reveals that this discontinuity corresponds to a shift from ECM 1 to 2 . As shown by the corresponding $\mathcal{I}(t)$, the ECL still oscillates periodically, and both the optical [Fig. 3(e2)] and RF spectra [Fig. 3(e1)] confirm that the frequency of the oscillations is still $7.5 \mathrm{GHz}$. The previous observations indicate that the trajectory moves in the positive-frequency direction and transits to another limit cycle, of the same frequency, on a different ECM. Increasing $\eta$ further, another limit cycle at $f_{\mathrm{RO}} \sim 7 \mathrm{GHz}$ is seen [region $\phi$ of Figs. 2(a), 2(b), 3(f1), and 3(f2)]. The active ECM 2 does not change in the transition from region $\varepsilon$ to $\phi$ of Fig. 2(a), indicating the existence of two different oscillation frequencies, 7.5 and $7 \mathrm{GHz}$, again separated by $\sim f_{\tau}$ on ECM 2 .

It must also be noted that to this point, a monotonic increase of $V_{\mathrm{LD}}$ is seen and $V_{\mathrm{LD}}$ is higher than for the initial condition (ECM 1) with $\eta \sim 0.02$. This indicates that the trajectory moves toward higher frequencies [right half in the $\Delta \phi$-vs- $N$ plane in Fig. 1 (b)]. The optical spectrum [Figs. 3(e2) and 3(f2)] also confirms that the dominant mode is ECM 2, which is at larger frequency. These observations are fundamental to understanding the route to chaos on the basis of the LK model. This confirms experimentally that the trajectory moves toward higher frequency at low $\eta$ for high $I$. Our experimental results agree with Masoller and Abraham [9], who calculated that initially, for small $\eta$, the dominant peak is shifted by a multiple of the ECM frequency spacing towards positive frequency. They attributed this behavior to the fact that ECMs that are close to the MLM (e.g., ECM -1 and -2) but of lower frequency have very small basins of attraction compared to positive ECMs at low $\eta$ for high $I$, while ECMs with large positive frequency shifts are highly unstable.

As $\eta$ is further increased, two sudden drops in $V_{\mathrm{LD}}$ are seen, one at $\eta \sim 0.38$ and the other at $\sim 0.45$. The values reached by $V_{\mathrm{LD}}$ are lower than the initial ECM 1 at $\eta=0.02$, indicating that the trajectory has abruptly moved toward ECMs at lower frequencies than the MLM [left in the $\Delta \phi$-vs- $N$ plane in Fig. 1(b)].

Figure $3(\mathrm{~g})$ shows the behavior of the ECL just after the second dropout [region $\iota$ of Figs. 2(a) and 2(b)]. The optical spectrum shows that the ECM is now near -10 [Fig. 3(g2)], whereas the RF spectrum highlights that the frequencies involved in the dynamics are $\sim 7$ and $3.5 \mathrm{GHz}$ [Fig. 3(g1)]. We interpret this region as a period-doubled limit cycle oscillation around ECM -10. It is interesting to note that other period-doubling observations reported in the literature $[18,19]$ correspond to the doubling of a period close to that of relaxations oscillations. Additionally, we interpret the 
previous region $\gamma$ of Figs. 2(a) and 2(b), as corresponding to a region where intermittency is observed between regions $\phi$ and $\gamma$ of Figs. 2(a) and 2(b). This interpretation is corroborated by the experimentally observed nonstationarity of ECL behavior in this region.

Finally, when $\eta$ exceeds $\sim 0.5$, thus reaching the maximum values of $\eta$ explored here, the trajectory wanders among several ECMs located at lower frequencies than the MLM, as indicated both by the optical spectrum and $V_{\mathrm{LD}}$. $\mathcal{I}(t)$ undergoes rapid chaotic fluctuations on the subnanosecond time scale [Fig. 3(h2)] and the broad RF spectrum [Fig. 3(h1)] indicates the excitation of a continuous range of frequencies. This regime is usually called in the literature fully developed coherence collapse (CC). This evolution of the laser dynamics around several negatively shifted ECMs is the typical prediction of the LK model under fully developed CC [1,3,9].

We have observed the main features of the BD presented in Fig. 2 for various large ranges of values of $L\left(f_{\tau} \ll f_{\mathrm{RO}}\right)$ and for values of $I \gtrsim 1.8 I_{\text {th }}$. When $I \lesssim 1.8 I_{\text {th }}$, qualitative changes occur as windows of stability corresponding to the settling of the trajectory on the MGM typically appear, as reported in Refs. [16,22].

In this work, we measured BDs of the optical intensity of an ECL with large $L$ and $I$. We observed that initial instabilities correspond to the first steps of a quasiperiodic route to chaos that develops around a single ECM. This ECM loses stability first via a Hopf bifurcation to limit-cycle behavior at a frequency close to $f_{\mathrm{RO}}$, which undergoes a secondary Hopf bifurcation corresponding to the undamping of a second frequency, close to $f_{\tau}$, and leading to quasiperiodic oscillations. The quasiperiodic route is then interrupted by various windows of periodicity, as well as by intermittent behavior. In addition, we observe the existence of several limit cycles of different frequencies around the same ECM as well as transitions between two different limit cycles existing around the same or two different neighboring ECMs. Finally, for larger $\eta$, the merging of numerous attractors corresponding to different ECMs dictates the dynamics which correspond to a chaotic region of fully developed CC. Our results provide detailed experimental observations of the bifurcation of a LD, biased well above $I_{t h}$, subjected to external optical feedback, and moreover provide detailed confirmation of some of the predictions of the LK model concerning the route to chaos for ECLs.

The authors acknowledge the financial support of the Conseil Régional de Lorraine.
[1] M. C. Soriano, J. García-Ojalvo, C. R. Mirasso, and I. Fischer, Rev. Mod. Phys. 85, 421 (2013).

[2] G. H. M. van Tartwijk and G. P. Agrawal, Prog. Quantum Electron. 22, 43 (1998).

[3] J. Ohtsubo, Semiconductor Lasers: Stability, Instability and Chaos (Springer, Berlin, 2006).

[4] M. Peil, I. Fischer, and W. Elsäßer, Phys. Rev. A 73, 023805 (2006).

[5] A. Argyris, D. Syvridis, L. Larger, V. Annovazzi-Lodi, P. Colet, I. Fischer, J. García-Ojalvo, C. R. Mirasso, L. Pesquera, and K. A. Shore, Nature (London) 438, 343 (2005).

[6] F.-Y. Lin and J.-M. Liu, IEEE J. Sel. Top. Quantum Electron. 10, 991 (2004).

[7] A. Uchida, K. Amano, M. Inoue, K. Hirano, S. Naito, H. Someya, I. Oowada, T. Kurashige, M. Shiki, S. Yoshimori, K. Yoshimura, and P. Davis, Nat. Photon. 2, 728 (2008).

[8] D. Brunner, M. C. Soriano, C. R. Mirasso, and I. Fischer, Nat. Commun. 4, 1364 (2013).

[9] C. Masoller and N. B. Abraham, Phys. Rev. A 57, 1313 (1998).

[10] J. Mork, J. Mark, and B. Tromborg, Phy. Rev. Lett. 65, 1999 (1990).

[11] D. Brunner, X. Porte, M. C. Soriano, and I. Fischer, Sci. Rep. 2, 732 (2012).

[12] T. Erneux and P. Glorieux, Laser Dynamics (Cambridge University Press, Cambridge, 2010).

[13] R. Lang and K. Kobayashi, IEEE J. Quantum Electron. 16, 347 (1980).

[14] J. Mork, B. Tromborg, and J. Mark, IEEE J. Quantum Electron. 28, 93 (1992).

[15] B. Tromborg and J. Mork, IEEE Photon. Technol. Lett. 2, 549 (1990).
[16] A. Hohl and A. Gavrielides, Phy. Rev. Lett. 82, 1148 (1999).

[17] H. Li, J. Ye, and J. G. McInerney, IEEE J. Quantum Electron. 29, 2421 (1993).

[18] J. Ye, H. Li, and J. G. McInerney, Phys. Rev. A 47, 2249 (1993).

[19] T. Mukai and K. Otsuka, Phys. Rev. Lett. 55, 1711 (1985).

[20] P. Berge, Y. Pomeau, and C. Vidal, Order within Chaos: Towards a Deterministic Approach to Turbulence (Wiley, Paris, 1986).

[21] B. Kim, A. Locquet, N. Li, D. Choi, and D. S. Citrin, IEEE J. Quantum Electron. 50, 965 (2014).

[22] B. Kim, N. Li, A. Locquet, and D. S. Citrin, Opt. Express 22, 2348 (2014).

[23] K. Peterman, IEEE J. Sel. Topics Quantum Electron. 1, 480 (1995).

[24] N. Li, B. Kim, A. Locquet, D. Choi, W. Pan, and D. S. Citrin, Opt. Lett. 39, 5949 (2014).

[25] C. H. Henry and R. F. Kazarinov, IEEE J. Quantum Electron. 22, 294 (1986).

[26] A. M. Levine, G. H. M. van Tartwijk, D. Lenstra, and T. Erneux, Phys. Rev. A 52, R3436 (1995).

[27] T. Heil, I. Fischer, and W. Elsasser, Phys. Rev. A 58, R2672 (1998).

[28] W. Ray, W. S. Lam, P. N. Guzdar, and R. Roy, Phys. Rev. E 73, 026219 (2006).

[29] A. A. Sahai, B. Kim, D. Choi, A. Locquet, and D. S. Citrin, Opt. Lett. 39, 5630 (2014).

[30] J. Ohtsubo, Opt. Rev. 6, 1 (1999).

[31] A. Ritter and H. Haug, IEEE J. Quantum Electron. 29, 1064 (1993).

[32] T. Erneux, Proc. SPIE 3944, 588 (2000).

[33] D. Pieroux, T. Erneux, T. Luzyanina, and K. Engelborghs, Phys. Rev. E 63, 036211 (2001). 